\section{Cattle help in conservation}

\author{
from Peter D. Moore
}

Although conservation has come to mean much more than the simple preservation of rare species, the prevention of extinction of threatened plants and animals is still an important concern of the conservationist. Apart from the possibility of aesthetic loss (consider how great a contribution the giant panda has made to the fantasy worlds of our children), a depletion of the planet's genetic resources accompanies each extinction which may ultimately be regretted even by those who have lost their aesthetic awareness. A good example of a lowly plant made rare by the destruction of suitable habitats and which has received such attention from conservationists is Dianthus armeria, the Deptford pink, which is restricted to a few meadows in southern Britain (see Walls, Proc. Bot. Soc. Br. Isl., 6, 337; 1967).

One of the sites at which this species occurs is Woodwalton Fen, a National Nature Reserve in the fenland area of East Anglia. Most of this nature reserve has been managed by man in the past, either for reed cutting, peat extraction or as grazing land; the area with Dianthus armeria was once a farmstead over which cattle were grazed. This practice was not continued in the early days of the reserve's history, but locally the pressure of rabbit grazing was sufficient to maintain the shortgrass community in which Dianthus survived. Following the myxomatosis epidemic of 1954, however, extensive changes occurred in the vegetation of the area; coarse grasses, particularly Calamagrostis epigejos and Agropyron repens, spread at the expense of shorter-growing species, including Dianthus. By 1964 only five plants remained and at this stage a new management regime involving grazing by Friesian heifers was introduced. Subsequently the popula- tion of Dianthus expanded gradually to 900 plants by 1967 .

Williams, Wells and Wells (J. appl. Ecol., 11, 499; 1974) have now attempted to analyse the respective roles of cattle and rabbit grazing in this particular recovery. They undertook a quantitative analysis of the faeces of these animals by concentrating the surviving plant epidermal fragments in a faecal sample and spreading them on a microscope slide. The species composition of these samples was subsequently estimated by means of randomly located point observations. This type of analysis was repeated periodically through the course of a year in order to determine the changing food preferences of the animals during that time. As Stewart (J. appl. Ecol., 4, 83 ; 1967) has pointed out, there are many problems associated with this technique, partioularly the relative digestibility of different species. But the preponderence of coarse grasses in the cattle faeces and the virtual absence of dicotyledonous species gives a reasonable representation of this animal's diet. The rabbits also seem to have neglected dicots, but show a stronger preference for the less coarse grasses, such as Festuca.

The results of these studies suggest that the beneficial effect of cattle upon the Dianthus armeria population results from their selective grazing upon the more aggressive species of grass. The techniques employed however, do not permit conclusions regarding the influence of trampling upon Dianthus by increasing microtopographical diversity. When scarce, the plant became restricted to ant hills, which suggests that soil disturbance and microtopographical factors may be important in controlling its distribution. Examination of the germination and establishment requirements of Dianthus under laboratory conditions might assist in the separation of these two distinct influences of cattle grazing. Meanwhile it cannot be denied that this is an efficient management practice for the conservation of a diminishing species.

discussion that these fears have been exaggerated.

Recent work on the genetic basis of Xeroderma Pigmentosum have shown the value of studies of heterokaryons formed by fusing fibroblasts from patients with the disease and from normal subjects. Dr P. Lohman (Netherlands Organisation for Applied Scientific Research, Rijswijk) reported that there are now five complementation groups in Xeroderma Pigmentosum. Dr Lohman also discussed experi-
The past few years have seen the emergence of Xeroderma Pigmentosum variants, patients with clinical features of the disease but with normal ability to carry out excision repair of DNA. Dr A. Lehmann (University of Sussex) has recently confirmed that in these patients all of the steps in excision repair are normal, but that there is a defect in post-replication repair. Post-replication repair in Xeroderma Pigmentosum variants is inhibited by caffeine, an effect which is not present in normal cells. Cells from patients with Xeroderma Pigmentosum who are defective in excision repair show changes in post-replication repair which are intermediate between normal and Xeroderma Pigmentosum variants.

Another aspect of this disease was discussed by Dr C. A. Ramsay (Institute of Dermatology, London). $\mathrm{He}$ demonstrated the abnormal reactions of the skin of these patients when they are irradiated with monochromatic ultraviolet light in the 290-320 nm region. The delay in the onset of erythema after irradiation may enable the diagnosis of Xeroderma Pigmentosum to be suspected early in life. In addition, Dr Ramsey reported the first successful case of the prenatal diagnosis of Xeroderma Pigmentosum by the detection of the deficiency of excision repair in cultured amniotic cells. Xeroderma Pigmentosum is the only condition to date in which excision repair of DNA has been found to be abnormal. Actinic keratoses occur on normal skin as a result of chronic exposure to solar ultraviolet irradiation and Dr J. Pitts (University of Glasgow) has found that in cells of such keratoses excision repair is not impaired.

Several other aspects of photosensitive skin disease were discussed. A review of the value of action spectroscopy in dermatology was given by Dr Frain-Bell (University of Dundee) The photodermatoses present many unsolved problems not least of which is that of treatment. Dr J. C. van der Leun (State University of Utrecht, Netherlands) has used the technique of repeated irradiation of the skin as a method of treatment and has found it of benefit in solar urticaria and polymorphic light eruption. These findings are of interest because the two diseases represent quite different reactions to sunlight; solar urticaria occurs very soon after irradiation whereas in polymorphic light eruption there is a delay of several hours before the skin changes occur.

Finally, a detailed study of the photochemical basis of a dye-induced photodermatitis was reported by $\mathrm{Dr} \mathrm{K}$. Davies (University of Salford). He showed that in this reaction with an antraquinone dye, singlet oxygen is an important intermediate. 